\title{
The Role of Cognitive Control in Tinnitus and Its Relation to Speech-in-Noise Performance
}

\author{
Yihsin Tai $^{1,2}$ and Fatima T. Husain ${ }^{1,2,3}$ \\ ${ }^{1}$ Department of Speech and Hearing Science, ${ }^{2}$ Beckman Institute for Advanced Science and Technology, ${ }^{3}$ Neuroscience Program, \\ University of Illinois at Urbana-Champaign, Urbana, IL, USA
}

\author{
Received August 31, 2018 \\ Revised September 20,2018 \\ Accepted September 27, 2018
}

\section{Address for correspondence Fatima T. Husain, $\mathrm{PhD}$ \\ Department of Speech and Hearing Science, Beckman Institute for Advanced Science and Technology, University of Illinois at Urbana-Champaign, 405 North Mathews Avenue, MC-251, Urbana, IL 61801, USA \\ Tel $+1-217-333-7561$ \\ Fax +1-217-333-2922 \\ E-mail husainf@illinois.edu}

Self-reported difficulties in speech-in-noise (SiN) recognition are common among tinnitus patients. Whereas hearing impairment that usually co-occurs with tinnitus can explain such difficulties, recent studies suggest that tinnitus patients with normal hearing sensitivity still show decreased SiN understanding, indicating that SiN difficulties cannot be solely attributed to changes in hearing sensitivity. In fact, cognitive control, which refers to a variety of top-down processes that human beings use to complete their daily tasks, has been shown to be critical for SiN recognition, as well as the key to understand cognitive inefficiencies caused by tinnitus. In this article, we review studies investigating the association between tinnitus and cognitive control using behavioral and brain imaging assessments, as well as those examining the effect of tinnitus on SiN recognition. In addition, three factors that can affect cognitive control in tinnitus patients, including hearing sensitivity, age, and severity of tinnitus, are discussed to elucidate the association among tinnitus, cognitive control, and SiN recognition. Although a possible central or cognitive involvement has always been postulated in the observed SiN impairments in tinnitus patients, there is as yet no direct evidence to underpin this assumption, as few studies have addressed both SiN performance and cognitive control in one tinnitus cohort. Future studies should aim at incorporating SiN tests with various subjective and objective methods that evaluate cognitive performance to better understand the relationship between $\mathrm{SiN}$ difficulties and cognitive control in tinnitus patients.

J Audiol Otol 2019;23(1):1-7

KEY WORDS: Tinnitus · Speech-in-noise recognition · Cognitive control of attention . Cognitive control of emotion.

\section{Introduction}

Tinnitus is a perception of sound without the presence of an external source. Tinnitus in a chronic form may affect emotional well-being (e.g., depression or anxiety), cause concentration difficulties and sleep disturbances, and thereby significantly impact an individual's quality of life [1].

Cognitive control refers to a variety of top-down processes that human beings use to complete daily tasks depending on their current goals, including but not limited to attention, memory, and processing speed [2]. The role of cognition in tinni-

This is an Open Access article distributed under the terms of the Creative Commons Attribution Non-Commercial License (https://creativecommons.org/licenses/by-nc/4.0/) which permits unrestricted non-commercial use, distribution, and reproduction in any medium, provided the original work is properly cited. tus has been discussed in several reviews [3,4], and articles outlining models (e.g., [5]). Moreover, the relation between tinnitus and cognitive control has been examined through behavioral tests, brain imaging techniques, and electrophysiological assessments. Increasing evidence suggests that cognitive abilities, such as attention, may be a prerequisite for optimal speech-in-speech processing in adverse listening environments $[2,6]$. Such abilities have also been reported to be the key to understanding cognitive inefficiency in tinnitus patients [7] and to further interpret speech comprehension difficulties reported in the tinnitus population [8]. The aim of this review is to discuss the association among tinnitus, cognitive control, and speech-in-noise (SiN) recognition. The review consists of three sections that address: 1) the relationship between cognitive control and $\mathrm{SiN}$ recognition, 2) behavioral 
and brain imaging evidence supporting changes of cognitive control in individuals with tinnitus, and 3) factors that affect cognitive control. Finally, the review ends by identifying gaps in knowledge that should be addressed by future research.

\section{Cognitive Control and Speech-in-Noise Recognition}

There is a consensus that SiN ability in the adult population can be attributed to individuals' peripheral hearing sensitivity, central auditory processing, and cognitive function [9]. Although hearing sensitivity can explain some of the SiN difficulties in listeners with hearing impairment, a growing body of evidence suggests that such difficulties are also present in those without hearing impairment, indicating that cognitive ability may be a more relevant driving factor to explain $\mathrm{SiN}$ difficulties [10]. In fact, an overall significant correlation of $\mathrm{r}=$ 0.31 between $\mathrm{SiN}$ recognition and cognitive performance, as well as significant associations between SiN recognition and various cognitive functions including inhibitory control, processing speed, and working memory, have been demonstrated through meta-analyses in individuals without tinnitus and with hearing sensitivity ranging from normal to moderate hearing loss [10].

To date, several studies on cognitive control, especially on attention, have suggested that individuals with chronic tinnitus tend to have difficulties in allocating their attentional resources [4]. Therefore, it may not be unusual to postulate that the effect of tinnitus on $\mathrm{SiN}$ performance can be observed in tasks that consume a significant amount of cognitive capacity, for example, speech recognition under multi-babble talkers.

\section{Speech-in-noise recognition in tinnitus}

A review of studies on speech comprehension from 1996 to 2016 suggests that tinnitus patients have poorer SiN recognition compared to hearing-matched controls, regardless of the heterogeneity of tinnitus population, hearing sensitivity, or the complexity of the SiN tasks [8]. In contrast, our recent study [11] did not support a general SiN deficit in tinnitus patients with normal hearing sensitivity, and both tinnitus and control groups showed similar SiN performance. Instead, our results indicated that the tinnitus group performed significantly worse when stimuli were presented monaurally to the left ear compared to the right ear, even though bilateral tinnitus percept and symmetrical hearing sensitivity were reported. Our findings suggest that the between-ear difference in tinnitus patients might not be solely interpreted by the structural differences in hemispheres of the brain, but a possible involvement of cognitive deficits that affect the between-ear SiN per- formance, because this right-ear advantage (or more specifically, left-ear disadvantage) has been shown to be modifiable by cognitive control such as attention [11]. In line with our assumption, Ivansic, et al. [8] indicated that most SiN studies in tinnitus suggest a central contribution to the observed speech comprehension difficulties. As to what might be involved in the so-called "central contribution," they linked it to the impaired cognitive functions observed in tinnitus patients and concluded that the effect of tinnitus may be particularly on attentional executive functions.

\section{Cognitive control of attention}

Attentional processing includes alerting attention (sustained alertness to process any incoming information), orienting attention (also called selective attention, which involves the conscious process of selecting task-relevant stimuli while ignoring task-irrelevant stimuli), and executive attention (top-down control of attention to resolve any conflicts of information) [12]. Although these networks can be addressed separately using various tasks, they often work together in real-world situations such as in SiN recognition [6].

Brain regions that involve the orienting and executive control of attention have been confirmed by brain imaging studies, the former consists of the dorsal and ventral attention system, and the latter includes the midline cortex as well as the anterior cingulate cortex [12]. These two networks have been extensively investigated behaviorally in the tinnitus population [3].

\section{Cognitive control of emotion}

Relevant to cognitive control of attention, cognitive control of emotion involves four processes: 1) the perception of a stimulus under the current context, 2) attention towards or away from the stimulus, 3) the significance of the stimulus is appraised based on its relevance to an individual's needs, and 4) response to the stimulus [13]. The regulation of emotion can be facilitated through various strategies such as attentional deployment (selective attention or distraction) towards a stimulus [13]. Taken together, the perception of tinnitus can impact both cognitive control of attention and emotion, and the changes in attention can thereby cause SiN difficulties (Fig. 1). In fact, converging evidence in brain imaging studies has confirmed the effect of tinnitus on non-auditory brain regions related to cognitive control [14].

Our interpretation of the interaction between cognitive control of attention and emotion and how they affect $\mathrm{SiN}$ in tinnitus differs from that proposed by Trevis, et al. (Fig. 1 in [15]), primarily in that they presume deficits in attention switching lead to persistence of tinnitus, whereas we are as- 


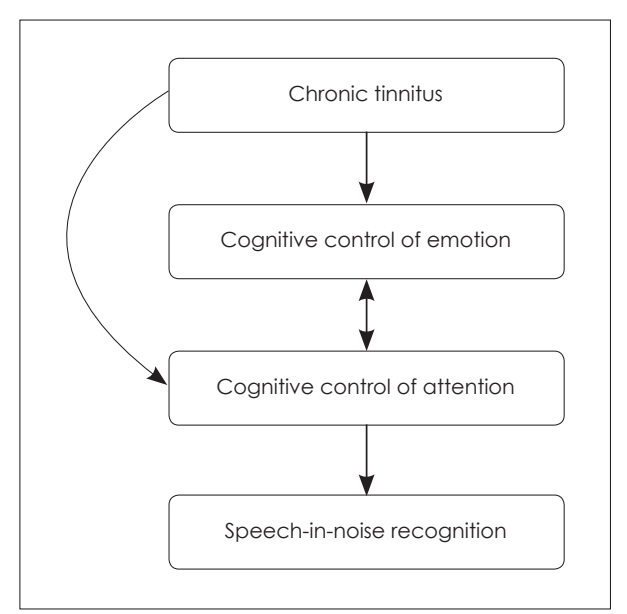

Fig. 1. Proposed association among chronic tinnitus, cognitive control, and speech-in-noise recognition.

suming such deficits to occur as tinnitus become chronic. Further, the severity of tinnitus may have a greater impact on cognitive control than merely on the perception of tinnitus, which differs from the Trevis, et al. framework [15].

\section{Changes in Cognitive Control in Tinnitus}

\section{Behavioral evidence}

Regardless of the methods being used, the relationship between tinnitus and cognitive control of attention has become a popular topic among tinnitus researchers. Roberts, et al. [5] suggest that auditory attention is key to either building a more accurate representation of the auditory scene when an unexpected auditory event occurs (in patients with non-bothersome tinnitus), or maintaining the scene when a mismatch of the top-down and bottom-up auditory information is present (in patients with bothersome tinnitus). Attention towards tinnitus can also be explained by the term "biased competition" [16], which specifies that sound streams that are more salient than others (e.g., louder in intensity or more distinct compared to others) can take place automatically. It should be noted that during daily listening tasks, tinnitus patients not only perceive their tinnitus but also external sounds such as speech or noise. Therefore, all listening tasks can be construed as dual-tasks due to the consumption of attentional capacity by the already existing tinnitus.

Several recent reviews have summarized studies on the effect of tinnitus on cognitive control of attention using behavioral or electrophysiological measures. For example, Mohamad, et al. [3] reviewed nine studies that showed behavioral evidence on changes in cognitive performance due to tinnitus, with a focus on the relation between tinnitus severity, work- ing memory, and various attentional networks. In their review, the theoretical construct of attention was precisely categorized by adopting the three major networks of the attention system described earlier [12]. Mohamad, et al. [3] concluded that tinnitus interferes with executive attention, but its effect on working memory and selective attention, as well as how the severity of tinnitus can impact these cognitive functions is uncertain. Moreover, they identified potential biases that need to be eliminated in future research; for example, an attempt to interpret the relation between tinnitus and cognitive functions without considering any confounding factors such as age and hearing impairment can lead to a selection bias.

A systematic review of 18 studies conducted by Tegg-Quinn, et al. [4] supplemented behavioral studies with those using electrophysiological measures to assess the impact of tinnitus on various cognitive functions. Their conclusion, similar to the one from Mohamad, et al. [3], was that tinnitus impacts cognitive function, specifically, the executive control of attention. They indicated that over time, studies on cognitive impairment in tinnitus patients gradually shifted their interpretation of the results from a non-specific deficit in cognitive function (i.e., a general deficit in attention) to a more refined one (a deficiency in executive control of attention). Additionally, they pointed out the problem of control groups not wellmatched on age or hearing sensitivity with the tinnitus group in several studies.

Further, studies using dual-task paradigms (Table 1), which involve executive attention as well as other functions of cognitive control (e.g., working memory), demonstrated that tinnitus patients show a depletion in attentional resources [7,17]. A dual-task paradigm requires individuals to perform two tasks simultaneously to examine the allocation of attentional capacity, under the assumption that the attentional capacity is limited. Generally, more attentional resources are needed to react to the increasing difficulty in the primary task, resulting in fewer attentional resources devoted to the secondary task. Longer reaction times and higher error rates in the secondary tasks may suggest a deficit in allocating the attentional resources. For example, Degeest, et al. [17] found that patients who reported a slight tinnitus handicap and normal hearing sensitivity still showed increasing listening effort, as manifested by a decrease in performance in their secondary task when completing speech recognition in quiet or in noise tests as the primary tasks.

In summary, increasing behavioral evidence corroborates the overarching conclusion that tinnitus can impact cognitive functions, especially the executive attention, which in turn can affect SiN. Additionally, the importance of eliminating confounding factors in studying cognitive functions in tinni- 
Table 1. Summary of behavioral studies discussed in this review

\begin{tabular}{|c|c|c|c|c|c|}
\hline \multirow[b]{2}{*}{ Study } & \multicolumn{3}{|c|}{ Tinnitus subjects } & \multirow{2}{*}{$\begin{array}{l}\text { Task(s) on cognitive } \\
\text { control of attention }\end{array}$} & \multirow{2}{*}{$\begin{array}{c}\text { Poorer performance } \\
\text { in tinnitus relative } \\
\text { to control group? }\end{array}$} \\
\hline & $\begin{array}{c}\text { Mean age }(S D) \text {, } \\
\text { range }\end{array}$ & $\begin{array}{l}\text { Tinnitus } \\
\text { severity }\end{array}$ & $\begin{array}{l}\text { Hearing } \\
\text { sensitivity }\end{array}$ & & \\
\hline Hallam, et al. [7] & $49.32(12), 20-70$ & - & $\mathrm{HL}$ & Dual-task paradigm & Yes \\
\hline Heeren, et al. [26] & 46.85 (15.79), $20-72$ & - & - & Attention Network Test & Yes \\
\hline Araneda, et al. [29] & $49(15.2), 20-67$ & $\begin{array}{l}\text { Moderate } \\
\qquad(\text { mean } \mathrm{THI}=43.29)\end{array}$ & $\mathrm{NH}+\mathrm{HL}$ & Go/no-go test & Yes \\
\hline Trevis, et al. [15] & $40.31(14.67), 18-60$ & $\begin{array}{l}\text { THI used, } \\
\text { score not reported }\end{array}$ & $\mathrm{NH}+\mathrm{HL}$ & N-back task; Stop-Signal task & Yes \\
\hline Degeest, et al. [17] & $23.8(4.3), 19-31$ & Slight (mean THI=12.2) & $\mathrm{NH}$ & Dual-task paradigm & Yes \\
\hline
\end{tabular}

THI: Tinnitus Handicap Inventory, $\mathrm{HL}$ : hearing loss, $\mathrm{NH}$ : normal hearing

tus is reiterated $[3,4]$. However, due to the heterogeneity of tinnitus population, it might not be possible to have an optimal match of both age and hearing sensitivity between the tinnitus and the control groups.

\section{Brain imaging evidence}

Our lab and others have used both task- and rest-based functional magnetic resonance imaging (fMRI) to investigate alterations in the neural network engaged in mediating attention. It should be pointed out that these studies do not demonstrate cognitive impairment in the tinnitus group when compared to the control group, but all describe alterations of the attention network. This may be either due to the type of tasks used in the fMRI studies or the type of tinnitus patients included, who predominantly reported mild symptoms.

In two studies $[18,19]$, we examined the activation patterns as a result of same-different task, involving either two or three stimuli, either auditory or visual. The auditory stimuli were pure tones of varying frequency while the visual stimuli were simple line drawing of the letters from the Korean alphabet (unfamiliar to the participants). In the first study [18], we observed reduced response in the parietal and frontal cortices for the tinnitus group relative to the hearing-matched control group (Fig. 2A) and decreased activation only in the frontal cortex compared to the normal hearing control group. In the second study [19], where we varied the load between two and three stimuli, the response of the fronto-parietal attention network was reduced in the tinnitus group compared to the control groups for both task loads of auditory stimuli (Fig. $2 \mathrm{~A}$ ), with the effect being more pronounced at high load. In contrast, in the visual modality, the tinnitus group exhibited greater response of the attention network, regardless of memory load, compared to the control groups (Fig. 2B). Thus, although there are alterations to the attention network due to chronic tinnitus, these appear to take the form of reduced engagement of the attention network for sounds and greater engagement for visual stimuli.
In an fMRI study of one-back task with distractors, using both auditory and visual stimuli, Amaral and Langers [20] found that although there were no group differences in terms of behavior, within the tinnitus group, performance worsened with severity. They also did not find differences in the neural activation of the central auditory or the dorsal attention system. Instead, they found greater engagement of the anterior insula and the cerebellum together with a less reduced deactivation of the visual cortex for the tasks for the tinnitus group compared to the control groups (Fig. 2B). The authors interpret these findings to suggest the altered engagement of the salience (due to the insular response) and the visual networks, inferring an abnormal response of the attentional control mechanisms in tinnitus.

With respect to cognitive control of emotion, a series of task-based studies have examined the effect of tinnitus severity on emotional processing $[21,22]$ and noted the increased response in the limbic system in those with bothersome tinnitus when processing affective stimuli compared to neutral stimuli (relative to a group with low distress). However, while in our study [21] we observed an increased response in the superior, middle and inferior frontal cortex in those with mild tinnitus when processing affective stimuli compared to neutral stimuli (relative to those with bothersome tinnitus), this finding was not supported in the Golm, et al. study [22], where they observed a decreased response in the middle frontal gyrus. This discrepancy may be related to the type of stimuli used in the studies (affective, every-day sounds in ours [21] and tinnitus-related and generally negative visually-presented sentences in Golm, et al. [22]).

Resting-state fMRI studies, where the participant does not perform a goal-directed task but is instructed to "rest and let the mind wander," also allow us to probe the functional connections of neural networks. As we have reviewed previously $[14,23]$, the main changes to neural networks appear to be decreased connectivity of the default mode network and increased engagement of the attention network at rest. Over time 

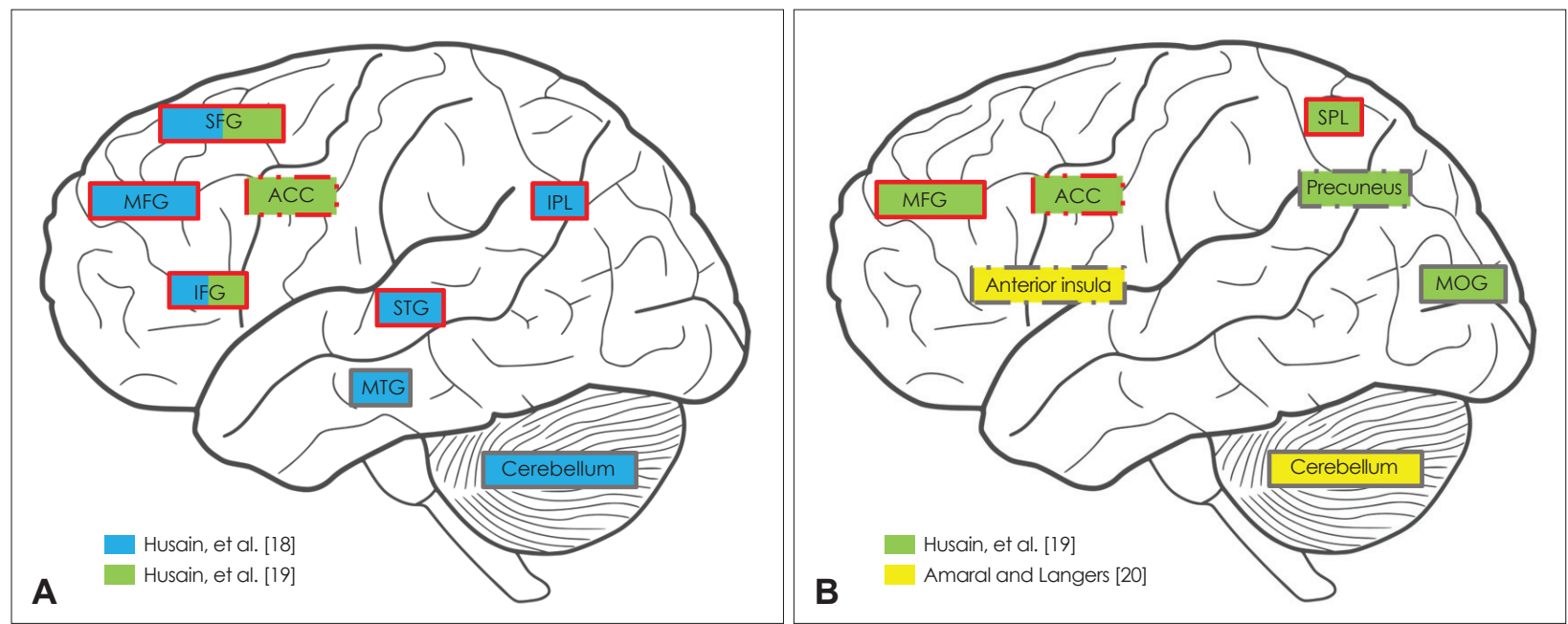

Fig. 2. Summary of findings in task-based functional magnetic resonance imaging studies on cognitive control of attention: brain regions with decreased activation during auditory tasks (A) and regions with increased activation during visual tasks (B) for the tinnitus group relative to the hearing-matched control group. Regions related to cognitive control of attention are marked with red frames and those that are in the medial brain are marked with dashed frames. SFG: superior frontal gyrus, MFG: middle frontal gyrus, IFG: inferior frontal gyrus, ACC: anterior cingulate cortex, STG: superior temporal gyrus, MTG: middle temporal gyrus, IPL: inferior parietal lobule, SPL: superior parietal lobule, MOG: middle occipital gyrus.

and as the severity of tinnitus increase, such alterations intensify [24].

\section{Factors Affecting Cognitive Control in Tinnitus}

\section{Hearing sensitivity}

A major problem in interpreting the findings of cognitive control in tinnitus patients is to parse out the impact of hearing loss, because tinnitus often co-occurs with hearing impairment [25]. However, it is not straightforward to evaluate the type and extent of hearing loss in the study participants, mostly because, what constitutes hearing loss or even normal hearing thresholds is variable between studies and hearing sensitivity is not evaluated or not reported (e.g., [26]). Such reasons can greatly prevent the readers from determining if any observed changes in cognitive control can be attributed only to the presence of tinnitus [4].

Although tinnitus without the presence of hearing impairment can still cause cognitive deficiencies [17], it is obvious that tinnitus accompanied by hearing loss demonstrates more consistent results in changes of cognitive control (e.g., for findings in brain imaging studies, [14]). In other words, it implies that cognitive control is highly susceptible to changes when tinnitus co-occurs with hearing loss, thereby making it more challenging to parse out the effect of hearing loss on cognitive control in tinnitus patients with hearing impairment.

\section{Aging}

In addition to hearing sensitivity, decreased cognitive control ability has been shown to be attributed to increasing age in the general population [2]. The effect of aging seems uncertain in the tinnitus population because both studies targeting young (e.g., Degeest, et al. [17]) and older tinnitus patients (e.g., Heeren, et al. [26]) have suggested altered cognitive control of attention caused by tinnitus behaviorally (Table 1). However, an insignificant effect of aging in this population may simply be due to the complexity of behavioral tasks, with more complex ones requiring greater effort of cognitive processing, regardless of the effect of aging. In fact, it might be impossible to preclude the effect of aging on cognitive control because most tinnitus studies included participants in a wide age range (e.g., tinnitus patients aged between 20 and 72 years in Heeren, et al. [26]). Moreover, unmatched age between the tinnitus and the control groups in some studies can adversely make the results susceptible to the effect of aging [4].

\section{Tinnitus severity}

The severity of tinnitus is often estimated by using established questionnaires such as the Tinnitus Handicap Inventory (THI) [27]. The THI has remained in widespread use because it provides five ranges of scores that allows the classification of various severity levels, despite being reported to have low sensitivity to smaller changes because it only contains a threelabel category scale. Questionnaires that contain subscales to reflect cognitive concerns and allow larger scale for rating, for example, the Tinnitus Primary Function Questionnaires [1] and 
the Tinnitus Functional Index [28], are considered to have better sensitivity. However, they have not been extensively used for studying cognitive control in tinnitus. Surprisingly, several studies (e.g., [7] and [26], Table 1) using behavioral measures to support changes in cognitive control of attention caused by tinnitus did not incorporate any tinnitus severity estimation.

It is difficult to predict the exact effect of tinnitus severity on cognitive control with the variety of tinnitus questionnaires used in estimating tinnitus severity and mixed findings from various studies. On the one hand, some patients who reported slight tinnitus handicap still showed difficulties in allocation attentional resource behaviorally [17]. On the other hand, other behavioral studies found altered cognitive control processing only in individuals who reported greater than slight tinnitus handicap (e.g., [29]).

Although fMRI studies have not often examined the role of severity on SiN or cognitive control, our recent paper examining resting state fMRI noted that changes to the connectivity patterns of the default mode and dorsal attention network were correlated with severity [24], thus attesting to the fact that severity has an impact even in the absence of a goal-directed task.

\section{Conclusion}

This review assessed the relationship between cognitive control and $\mathrm{SiN}$ performance in tinnitus patients. In general, SiN studies suggest an impact of tinnitus on SiN performance, with poorer $\mathrm{SiN}$ recognition observed in patients compared to controls. Such findings appear to be independent of hearing sensitivity or the complexity of SiN tasks, but the effect of tinnitus severity cannot be ruled out. In terms of cognitive control in tinnitus, studies have demonstrated that tinnitus can alter cognitive control processing, but confounding factors such as hearing sensitivity and tinnitus severity need to be considered for a complete interpretation of results. Because few studies have addressed both $\mathrm{SiN}$ recognition and cognitive control in tinnitus patients, the evidence is still not welldefined as to how cognitive control is affected by tinnitus and how tinnitus indirectly affects $\mathrm{SiN}$ performance by causing cognitive deficiencies.

\section{Future directions}

To better understand how cognitive control can impact SiN performance in tinnitus patients, future $\mathrm{SiN}$ studies should aim at incorporating both cognitive tasks and other methods to examine changes in cognitive control (e.g., event-related potentials or fMRI) in a cohort with tinnitus. Moreover, it might be informative to use self-reported questionnaires to in- vestigate if tinnitus patients with normal hearing sensitivity truly have concerns regarding speech recognition in adverse listening environments. Additionally, with increasing number of tinnitus studies, meta-analyses of studies using comparable methods with various age groups and hearing profiles can be advantageous in delineating an overarching conclusion on the association among tinnitus, cognitive control, and SiN performance.

In conclusion, deficits in cognitive control may be related to concentration or communication difficulties when processing auditory stimuli (e.g., speech recognition in noise) reported by tinnitus patients regardless of their hearing status. Future investigation in tinnitus patients incorporating a range of ages and hearing profiles can be invaluable in assessing the effectiveness of clinical tinnitus intervention and management, in monitoring the progression of tinnitus (e.g., through comparing recent-onset to chronic tinnitus, or through conducting longitudinal studies), and may also play a key role in developing patient-specific treatment strategies.

\section{Acknowledgments}

We wish to thank Rafay Khan for his comments on earlier drafts of the manuscript. Both authors were supported by the US Department of Defense grant W81XWH-15-2-0032 while writing the review.

\section{Conflicts of interest}

The authors have no financial conflicts of interest.

\section{REFERENCES}

1) Tyler R, Ji H, Perreau A, Witt S, Noble W, Coelho C. Development and validation of the tinnitus primary function questionnaire. Am $\mathrm{J}$ Audiol 2014;23:260-72.

2) Moore DR, Edmondson-Jones M, Dawes P, Fortnum H, McCormack A, Pierzycki RH, et al. Relation between speech-in-noise threshold, hearing loss and cognition from 40-69 years of age. PLoS One 2014;9:e107720.

3) Mohamad N, Hoare DJ, Hall DA. The consequences of tinnitus and tinnitus severity on cognition: a review of the behavioural evidence. Hear Res 2016;332:199-209.

4) Tegg-Quinn S, Bennett RJ, Eikelboom RH, Baguley DM. The impact of tinnitus upon cognition in adults: a systematic review. Int $\mathrm{J}$ Audiol 2016;55:533-40.

5) Roberts LE, Husain FT, Eggermont JJ. Role of attention in the generation and modulation of tinnitus. Neurosci Biobehav Rev 2013;37: 1754-73.

6) Valderrama JT, Beach EF, Yeend I, Sharma M, Van Dun B, Dillon H. Effects of lifetime noise exposure on the middle-age human auditory brainstem response, tinnitus and speech-in-noise intelligibility. Hear Res 2018;365:36-48.

7) Hallam RS, Mckenna L, Shurlock L. Tinnitus impairs cognitive efficiency. Int J Audiol 2004;43:218-26.

8) Ivansic D, Guntinas-Lichius O, Müller B, Volk GF, Schneider G, Dobel C. Impairments of speech comprehension in patients with tinnitus-a review. Front Aging Neurosci 2017;9:224.

9) Akeroyd MA. Are individual differences in speech reception related to individual differences in cognitive ability? A survey of twenty experimental studies with normal and hearing-impaired adults. Int J 
Audiol 2008;47 Suppl 2:S53-71.

10) Dryden A, Allen HA, Henshaw H, Heinrich A. The association between cognitive performance and speech-in-noise perception for adult listeners: a systematic literature review and meta-analysis. Trends Hear 2017;21:2331216517744675.

11) Tai Y, Husain FT. Right-ear advantage for speech-in-noise recognition in patients with nonlateralized tinnitus and normal hearing sensitivity. J Assoc Res Otolaryngol 2018;19:211-21.

12) Petersen SE, Posner MI. The attention system of the human brain: 20 years after. Annu Rev Neurosci 2012;35:73-89.

13) Ochsner KN, Silvers JA, Buhle JT. Functional imaging studies of emotion regulation: a synthetic review and evolving model of the cognitive control of emotion. Ann N Y Acad Sci 2012;1251:E1-24.

14) Husain FT. Neural networks of tinnitus in humans: elucidating severity and habituation. Hear Res 2016;334:37-48.

15) Trevis KJ, McLachlan NM, Wilson SJ. Cognitive mechanisms in chronic tinnitus: psychological markers of a failure to switch attention. Front Psychol 2016;7:1262.

16) Shinn-Cunningham BG. Object-based auditory and visual attention. Trends Cogn Sci 2008;12:182-6.

17) Degeest S, Keppler H, Corthals P. The effect of tinnitus on listening effort in normal-hearing young adults: a preliminary study. J Speech Lang Hear Res 2017;60:1036-45.

18) Husain FT, Pajor NM, Smith JF, Kim HJ, Rudy S, Zalewski C, et al. Discrimination task reveals differences in neural bases of tinnitus and hearing impairment. PLoS One 2011;6:e26639.

19) Husain FT, Akrofi K, Carpenter-Thompson JR, Schmidt SA. Alterations to the attention system in adults with tinnitus are modality specific. Brain Res 2015;1620:81-97.

20) Amaral AA, Langers DR. Tinnitus-related abnormalities in visual and salience networks during a one-back task with distractors. Hear Res 2015;326:15-29.

21) Carpenter-Thompson JR, Schmidt S, McAuley E, Husain FT. Increased frontal response may underlie decreased tinnitus severity. PLoS One 2015:10:e144419.

22) Golm D, Schmidt-Samoa C, Dechent P, Kröner-Herwig B. Neural correlates of tinnitus related distress: an fMRI-study. Hear Res 2013; 295:87-99.

23) Husain FT, Schmidt SA. Using resting state functional connectivity to unravel networks of tinnitus. Hear Res 2014;307:153-62.

24) Schmidt SA, Carpenter-Thompson J, Husain FT. Connectivity of precuneus to the default mode and dorsal attention networks: a possible invariant marker of long-term tinnitus. Neuroimage Clin 2017; 16:196-204.

25) Møller AR. Sensorineural tinnitus: its pathology and probable therapies. Int J Otolaryngol 2016;2016:2830157.

26) Heeren A, Maurage P, Perrot H, De Volder A, Renier L, Araneda R, et al. Tinnitus specifically alters the top-down executive control subcomponent of attention: evidence from the Attention Network Task. Behav Brain Res 2014;269:147-54

27) Newman CW, Jacobson GP, Spitzer JB. Development of the tinnitus handicap inventory. Arch Otolaryngol Head Neck Surg 1996;122: 143-8.

28) Meikle MB, Henry JA, Griest SE, Stewart BJ, Abrams HB, McArdle $\mathrm{R}$, et al. The tinnitus functional index: development of a new clinical measure for chronic, intrusive tinnitus. Ear Hear 2012;33:153-76.

29) Araneda R, De Volder AG, Deggouj N, Renier L. Altered inhibitory control and increased sensitivity to cross-modal interference in tinnitus during auditory and visual tasks. PLoS One 2015;10:e0120387. 